\title{
SUR LE GENRE RICTULARIA FROELICH 1802 (NÉMATODES SPIRUROIDEA)
}

\section{Par Robert Ph, DOLLFUS et Camille DESPORTES}

I. - Généralités. Caractères de discrimination des espèces. Cycle évolutif

Dans la superfamille des Spiruroidea A. Railliet et A. Henry 1915, la famille des Rictulariidæ A. Railliet 1916 a été ramenée au rang de sous-famille (Rictulariinæ M.-C. Hall 1913) et celle-ci est maintenant placée par B.-G. Chitwood et E.-E. Wehr (1934, p. 313), avec quatre autres sous-familles, dans la famille des Thelaziidæ A. Railliet 1916, alors que, primitivement, M.-C. Hall (1913, p. 75) avait considéré les Rictulariinæ comme sous-famille de Metastrongylidæ A. Railliet et A. Henry 1910. Selon H.-A. Baylis $(1928$, p. 301 ; 1934, p. 349), Rictularia se plaçait dans la famille des Spiruridæ et la sous-famille des Thelaziinæ.

Pour les Rictulariina, la définition suivante a été donnée par B.-G. Chitwood et E.-E. Wehr (1934, p. 317) :

Thelaziida : Ouverture orale généralement déplacée vers la face dorsale, le degré de déplacement variant avec l'espèce. Lèvres et pseudolèvres absentes. Papilles du cercle interne distinctes, avec terminaison poriforme ; papilles dorso-dorsales et ventro-ventrales du cercle externe petites, situées près des papilles latéro-dorsales et latéro-ventrales. Cuticule armée de crochets ou épines; bosses cuticulaires absentes. Ailes caudales présentes ou absentes. Spicules égaux ou inégaux. Gubernaculum présent. Vulve prééquatoriale ou équatoriale. Ovipares.

Genre type : Rictularia Frœlich 1802. Autres genres : Pneumonema H.-T. Johnston 1916, Echinonema Linstow 1898, Rictularioides M.-C. Hall 1916.

Ces quatre genres se distinguent facilement d'après les caractères indiqués dans la clef :

1. Epines disposées par rangées transversales ......... Echinonema Epines disposées par files longitudinales $\ldots \ldots \ldots \ldots \ldots \ldots \ldots .2$

ANn. de Parasitologie, T. XX, ${ }^{\circ s} 1-2,1944-1945$, p. 6-34. 
2. Deux files longitudinales d'épines de chaque côté du corps.Pneumonema Deux ou trois files longitudinales à la face ventrale $\ldots \ldots \ldots \ldots \ldots 3$

3. Deux files subventrales d'épines (1) ............. Rictularia Trois files subventrales d'épines ............. Rictularioides

Le genre Spinitectus Fourment 1883, qui avait été compris dans les Rictulariinæ par W. Yorke et P.-A. Maplestone (1926, p. 344), en a été éliminé par B.-G. Chitwood et E.-E. Wehr (1934, p. 317, 320) qui l'ont placé dans les Spirurinæ, à côté d'Ascarophis P.-J. Van Beneden 1870.

Dans le genre Rictularia, tel qu'il a été ąctuellement délimité, sont placées au moins 30 espèces, dont beaucoup sont très insuffisamment décrites et figurées ; il est fort probable que l'on a considéré comme appartenant à des espèces séparées, des formes qui sont en réalité conspécifiques (par exemple tani et whartoni) et il est vraisemblable que l'on a rapporté des formes qui ne sont apparemment pas conspécifiques, à une même espèce (par exemple spinosa et plagiostoma).

Il est difficile de classer les Rictulaires à la fois d'après leur morphologie et d'après l'hôte parasité.

L.-A. Jägerskiöld (1909, p. 43), M.-C. Hall (1913, p. 77, 81), L.-G. Seurat $(1916$, p. 147, 149) ont estimé que l'on pouvait distinguer deux groupes d'espèces, l'un chez les Carnivores, l'autre chez les Rongeurs, Chéiroptères, Insectivores. D'après Hall, dans le groupe des espèces parasites des Carnivores (cahirensis, affinis, splendida), chez la femelle, la transition entre les « crêtes pectiniformes » et les épines est très graduelle et a lieu assez loin en arrière de la vulve ; chez le mâle, ces crêtes latéro-ventrales s'étendent pòstérieurement jusqu'au cloaque ou presque. Dans le groupe des espèces parasites de Rongeurs, Chéiroptères, Insectivores, chez la femelle la transition entre les crêtes pectiniformes et les épines est rapide et nette (2) : elle a lieu au niveau de la vulve ; chez le mâle, ces crêtes cessent, postérieurement, avant le cloaque.

(1) Chez le mâle, il peut $y$ avoir quelques peignes médio-ventraux précloacaux; ils ne sont pas considérés comme une troisième file longitudinale ventrale.

(2) Cependant, chez fallax, la transition entre peignes et épines est plutôt graduelle et a lieu assez loin en arrière de la vulve. Si la transition est brusque chez scalopis, elle est ménagée chez proni; elle est nette évidemment chez jögerskiöldi, tani, macdonaldi, tandis que chez bovieri la transition est insensible et débute bien en avant de la vulve. Chez alphi (de Primates), il y a un passage brusque de peignes hauts à des peignes bas au niveau de l'œsophage et les peignes atteignent l'extrémité du corps (contrairement à ce qui a lieu chez eivirie, cristata, amurensis, par exemple).

Le niveau et le mode de transition ne donnent donc pas de caractères suffsants pour permettre de répartir les espèces en groupes homogènes, mais ce sont de bons caractères de discrimination spécifique. 
Pour Seurat (1916, p. 147), le groupe des Rictulaires de Rongeurs comprend une série de formes caractérisées "par la brièveté de l'ovéjecteur, la petite taille du mâle, l'absence d'ailes caudales, l'existence de papilles génitales sessiles et l'inégalité des spicules 》; dans ce groupe, qui comprend, selon Seurat, plagiostoma, fallax, cristata, macdonaldi, il range évidemment proni ; mais proni a été trouvé, dans la même localité, à la fois chez un Carnivore (Herpestes) et chez un Rongeur (Arvicanthis) ; cela oblige Seurat (1916, p. 147 ; 1917, p. 24) à considérer Arvicanthis comme l'hôte normal de proni, qui serait seulement "égaré 》 chez Herpestes. Remarquons aussi que Seurat $(1915$, p. $320 ; 1917$, p. 24$)$ a rapporté à macdonaldi, dont l'hôte type est un Chéiroptère (Megaderma), un spécimen mâle récolté chez un Carnivore (Genetta) ; il faudrait donc admettre, dans ce cas aussi, que cet exemplaire était égaré chez la Genette, mais on peut aussi se demander s'il s'agissait bien de macdonaldi.

L'inégalité des spicules est-elle un caractère commun aux espèces non parasites de Carnivores ? Cela n'est plus soutenable actuellement: les spicules sont égaux chez affinis, cahirensis, splendida, vulpis, houdemeri, mais ils le sont aussi chez citelli et harrisi qui n'ont jamais été trouvés chez des Carnivores; ils sont subégaux chez tani, des Rats (cf. H.-T. Chen, 1936, p. 283) ; ils sont généralement égaux, mais parfois très inégaux, chez alphi, des Primates; enfin ils sont inégaux chez coloradensis, elegans, halli, macdonaldi, mjöbcrgi, muris, proni, taterilli.

Divers autres caractères (dents buccales, papilles céphaliques, papilles caudales, crêtes médianes flabelliformes précloacales du mâle, etc...) sont utilisés pour la discrimination des espèces ; toutefois l'on n'est pas arrivé, jusqu'à présent, à séparer les espèces en groupes naturels homogènes, où des caractères morphologiques communs seraient en liaison avec une distribution géographique particulière ou avec le groupe d'hôtes parasités.

Par exemple, les Rictulaires des Sciuroidea ne se distinguent pas de tous les autres par des caractères qui leur appartiennent en propre, les Rictularia paléarctiques n'ont pas en commun des caractères particuliers les opposant aux Rictularia néarctiques, etc...

Pour l'identification des femelles, il est important de connaître le niveau de la vulve par rapport à l'extrémité de l'œsophage et par rapport aux paires d'épines pectiniformes: il faut toutefois tenir compte des variations individuelles, il s'en faut que ce niveau soit absolument constant.

La vulve est antérieure à l'extrémité postérieure de l'œsophage chez: alphi, cancasica, macdonaldi, proni, taterilli, sp. (in Esthosciu- 
rus); elle est au niveau de l'extrémité postérieure de l'œsophage chez coloradensis; au même niveau ou un peu en avant ou un peu en arrière chez affinis (cf. Jägerskiöld, 1909, p. 19-20 ; L. Seurat, 1915, p. 318 ; Sandground, 1933, p. 292), chez harrisi et chez whartoni ; elle est postérieure (plus ou moins) à l'extrémité postérieure de l'œsophage chez æthechini, cristata, fallax, cahirensis, halli, houdemeri, jägerskiöldi, amurensis, magna, mjöbergi, plagiostoma, scalopis, splendida, wheeleri, paradoxuri.

Le niveau de la vulve par rapport aux paires d'épines pectiniformes est indiqué dans le tableau ci-après (1).

Dans ce tableau, nous avons mentionné séparément proni Seurat sensu et proni Gendre sensu, en raison de la grande différence de niveau de la vulve par rapport aux paires d'épines pectiniformes ; nous ignorons cependant s'il s'agit ou non de la même espèce. Nous n'avons pas considéré, malgré Willemoes-Suhm, spinosa comme identique à plagiostoma. La synonymie de mjöbergi, paradoxuri et wheeleri parait probable.

Des clefs de détermination ont été données pour une partie des espèces du genre par L.-A. Jägerskiöld (1909, p. 43), M.-C. Hall (1913, p. 81 ; 1916, p. 169), M.-P. Lubimov (1933, p. 258-260) ; leur utilisation est malheureusement très limitée.

Cycle évolutif. - D'après le régime alimentaire des hôtes, dont plusieurs sont exclusivement entomophages, l'on suppose que la larve des Rictulaires se trouve chez des-Insectes. Cependant G. Witenberg $(1928$, p. 603), à Jérusalem, a obtenu Rictularia cahirensis Jägersk. expérimentalement chez de jeunes chiens à qui il avait fait ingérer des viscères de Reptiles dont les séreuses étaient contaminées par des kystes vermineux. Dans l'intestin de l'hôte définitif, Witenberg constata que deux mues se produisaient avant l'achèvement du développement. Vraisemblablement ces Reptiles n'étaient, admet Witenberg, que deuxième hôte intermédiaire ; il est en effet probable que les larves de Rictularia évoluent d'abord chez des Insectes coprophages et que ceux-ci sont ingérés par des Reptiles.

Certaines espèces, tout ati moins, peuvent devenir adultes dans des hôtes qui ne sont certainement pas ceux où ils évoluent normalement.

(1) Nous considérons le nombre des épines pectiniformes prévulvaires comme le plus important, car il est facile de l'observer exactement, tandis que le nombre des épines post-vulvaires risque d'ètre indiqué avec moins de précision quand il s'agit d'espèces où, postérieurement, les épines diminuent de taille, s'estompent, s'espacent et se raréfient. 


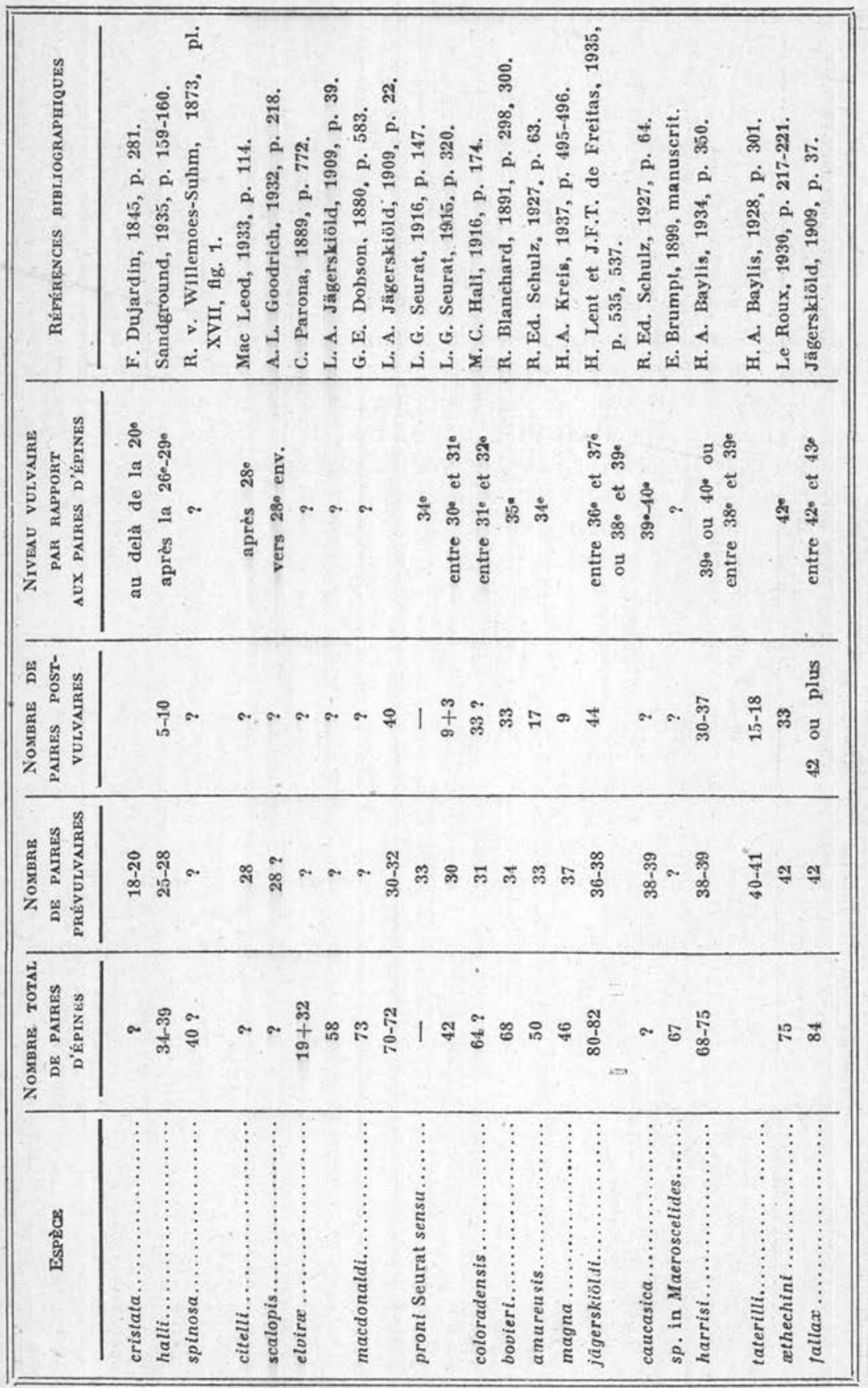




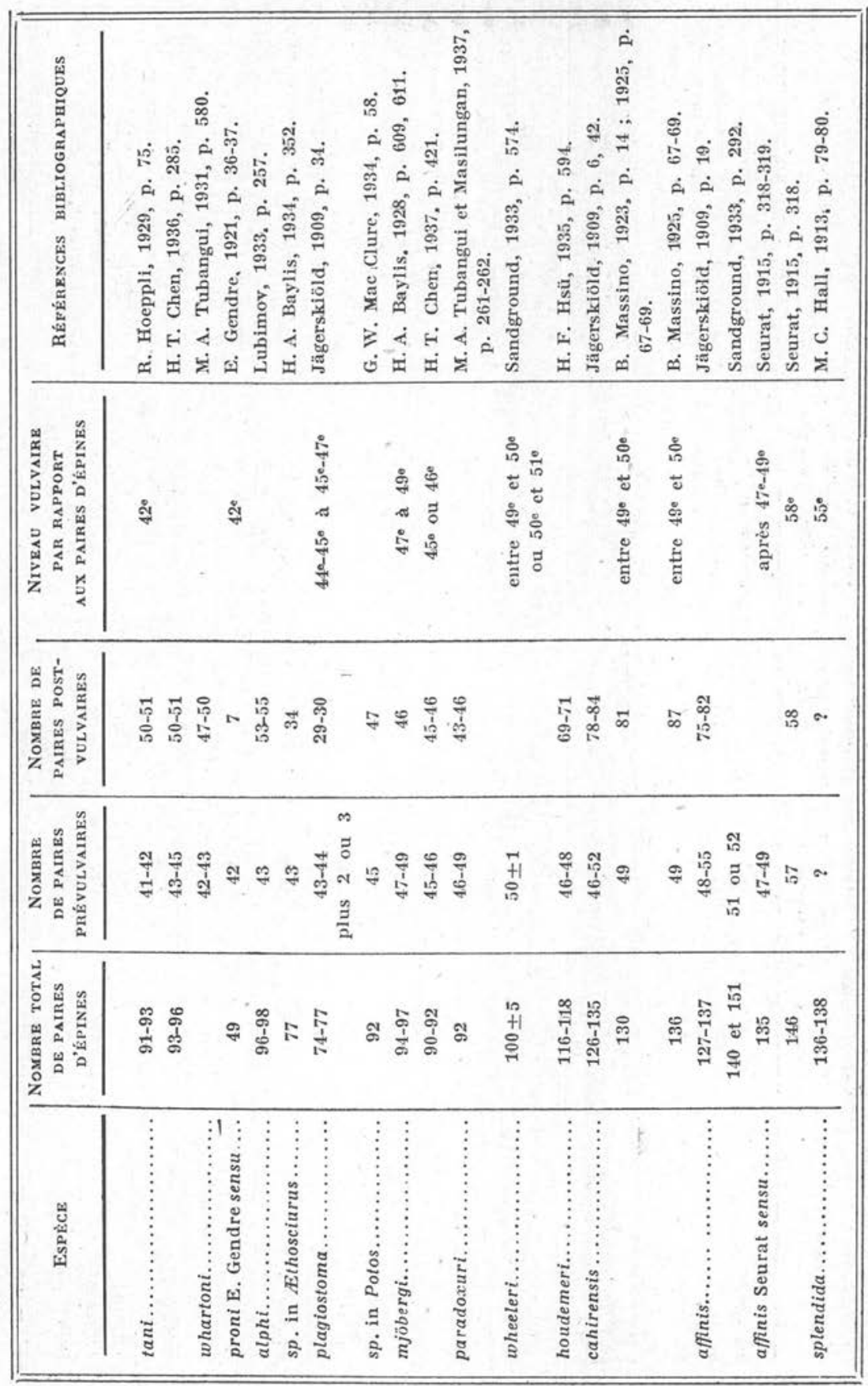




\section{II. - Rictularia cristata I.-A. Frœlich 1802, p. $9-13,88$; pl. I, fig. $1-3$}

La morphologie de cette espèce est encore très peu connue. Il n'existe que deux descriptions: celle de Frolich et celle de Dujardin.

Frolich, travaillant à Elwangen (Allemagne), trouva quelques jeunes femelles (longues d'env, 23,7 à $28 \mathrm{~mm}$.) dans l'intestin grêle d'un Rongeur qu'il rapporta alors inexactement à "Mus silvaticus Linné », alors qu'il s'agissait en réalité de Myoxus avellanarius (L.) (= Myoxus muscardinus Schreber) ainsi qu'il en avertit Bremser en une lettre dont fit mention Rudolphi (1819, p. 304).

La description et les figures originales sont peu détaillées et certainement pas bien exactes. Frœlich conserva ses vers vivants pendant cinq jours ; il nota (1802, p. 10) que les épines pectinées étaient mobiles, pouvant " selon la volonté du ver » tantôt s'étendre, tantôt se rabattre "comme les rayons dorsaux des poissons ». Le nombre des épines pectinées par files longitudinales n'a pas été précisé par Frœlich ; d'après sa figure 1, il pourrait y en avoir 22 prévulvaires ; d'après le texte, il pourrait $\mathrm{y}$ en avoir quelques-unes au delà.

F. Dujardin (1845, p. 280-281) récolta ses spécimens dans le duodénum de deux mulots Apodemus sylvaticus (L.), à dix kilomètres au nord de Rennes; il ne trouva que des femelles : les unes adultes, longues de 40 à $66 \mathrm{~mm}$., les autres immatures, longues de $16 \mathrm{~mm}$. Dujardin n'a vu d'épines pectinées que jusqu'à la vulve ; il en a compté 18 à 20 par file longitudinale. Les œufs múrs, pourvus de deux coques et contenant un embryon replié, mesuraient $50 \mu$.

R. cristata Frol. a aussi été trouvé au Musée de Vienne : une fois chez Eliomys quercinus (L.) (= Myoxus dryas Schreber) et quatre fois sur trente-deux chez Glis glis (L.), d'après ce que rapportent Rudolphi (1819, p. 61, 304) et Dujardin (1845, p. 281).

Ces spécimens du Musée de Vienne n'ont pas été utilisés pour une nouvelle description, cependant ils ont dû être examinés par Diesing, car celui-ci $(1851$, p. 245$)$ dit que, selon lui, les individus trouvés chez glis et signalés comme «Ophiostoma cristata 》 par Rudolphi, appartiennent à une espèce particulière autre que celle de Frœlich.

Aux environs de Tiflis (Caucase), J.-D. Kirschenblatt (1938, p. 44, $71,72,85)$ a trouvé quelques femelles de Rictularia dans l'intestin grêle d'un Apodemus sylvaticus (L.) et de deux Mus musculus abbotti Waterhouse ; il n'a pas décrit son matériel ; il l'a seulement rapporté à cristata, rappelant que l'espèce est caractérisée par la présence de crêtes (ou épines pectinées) sur un seul côté du corps. 
Kirschenblatt n'a pas dit s'il avait vérifié ce caractère : cela aurait eu un grand intérêt, car l'on admet généralement que cristata a bien deux files de peignes, comme les autres espèces du genre et que Froelich a mal interprété ses préparations.

Il n'est pas possible, aujourd'hui, de donner une bonne description de cristata; il faudrait revoir les spécimens du Musée de Vienne ét ceux de Kirchenblatt; nous ignorons si ceux de Frœlich existent encore ; ceux de Dujardin ont disparu comme toute la collection helminthologique de Dujardin.

Nous avions cru avoir retrouvé cristata chez A podemus sylvaticus (L.), à Richelieu (Indre-et-Loire) (28 août 1932), mais l'étude de nos spécimens n'a pas confirmé cette identification ; selon nous, cristata, tant qu'il n'aura pas été redécrit, est species inquirenda.

\section{III. - Matériel récolté a Richelied (INdRE-et-Loire) CHEZ Sciurus vulgaris ET Apodemus sylvaticus}

Notre matériel, récolté à Richelieu (Indre-et-Loire), provient :

$1^{\circ}$ d'un Mulot [Apodemus sylvaticus (L.)], 28 août 1932 : cinq femelles dans l'intestin ;

$2^{\circ}$ de deux Ecureuils [Sciurus vulgaris L.] (14 juillet 1932 et 12 août 1932) : deux femelles dans le cæcum chez le premier Ecureuil, une femelle dans l'estomac du second; au cours des années suivantes, aucun Rictularia ne fut retrouvé, tant chez des Ecureuils que chez des Mulots.

Nous avons eu, en tout, neuf femelles à notre disposition : de leur étude, il ressort qu'il s'agit de la même espèce chez le Mulot et chez les deux Ecureuils.

\section{A. Individus récoltés chez Sciurus}

Corps long de $48 \mathrm{~mm}$. 6 à $50 \mathrm{~mm}$, large dans sa partie moyenne (alors qu'aucune pression n'est exercée à sa surface) de $1 \mathrm{~mm}$. env. ; progressivement aminci vers l'extrémité antérieure, peu atténué postérieurement.

Cuticule épaisse $(18 \mu)$, élargie dans la région cervicale (75 à $90 \mu)$ et formant deux expansions latérales aliformes, présentant quelques stries longitudinales (au-dessus des bandes musculaires) et des stries transversales très irrégulières.

Extrémité céphalique arrondie, avec une bouche franchement dorsale, limitée par deux fausses lèvres, dorsale et ventrale, peu épaisses, appliquées contre la capsule buccale. 
Capsule buccale complète, fortement chitinisée en avant (partie ventrale), où elle est concave, et en arrière (partie dorsale), où elle est un peu convexe ; plus mince et moins élevée sur les côtés ; garnie sur son bord libre de 28 à 32 denticules (12 à 16 pour le bord ventral et 12 à 16 pour le bord dorsal). Les denticules les plus élevés sont situés au milieu des bords antérieur et postérieur; toutefois, ceux du bord antérieur (formant une sorte de mâchoire ventrale) sont souvent répartis en trois groupes assez distincts; plus rarement, on observe quatre de ces groupes.

La partie profonde de la capsule buccale est bordée intérieurement d'un bourrelet saillant qui suit plus ou moins le contour de la lumière œsophagienne et se relève au-dessus des trois portions de l'œsophage sous forme de crêtes qui simulent trois dents épaisses et émoussées à leur sommet : celle de ces dents qui occupe la position dorsale est alors beaucoup plus élevée que les deux autres, subventrales.

Papilles céphaliques : elles sont au nombre de cinq paires (1), dont trois se terminent par les curieuses formations en croissant mentionnées par différents auteurs [Jägerskiöld (1909, pl. II, fig. 1), Schulz (1927), Baylis (1928), Le Roux (1930), Sandground (1933), etc...] ; ces trois paires de papilles font partie du cercle externe, qui est déplacé par la translation de la bouche : une première paire, médioventrale, est tout à fait antérieure et ses formations en croissant, droit et gauche, presque contiguës; la seconde, latérale, est très importante ; la troisième médiodorsale, beaucoup plus petite, est située sur la marge de la lèvre dorsale. Les deux autres paires de papilles - qui représentent les latéro-médiales du cycle externe sont hémisphériques, mais à sommet légèrement affaissé antérieurement : une paire de ces papilles, latéro-dorsale, se trouve au voisinage de la lèvre dorsale (cette paire semble considérée par Sandground, chez Rictularia halli, comme représentant les amphides); l'autre, morphologiquement identique à la précédente, est latéro-ventrale. Cette dernière paire ne semble avoir été observée que chez $R$. amurensis par R.-Ed. Schulz (1927). Les pores amphidiaux s'ouvrent légèrement en arrière et à la base des papilles latérales. Le cycle interne, très réduit, n'est pas apparent.

Il existe, en outre, un pli cuticulaire transversal important en arrière de la lèvre dorsale et différents reliefs céphaliques sous-cuticulaires, représentant les terminaisons des bandes musculaires.

Epines cuticulaires, caractéristiques du genre, disposées en deux

(1) Les papilles submédianes sont franchement dédoublées chez Rictularia. 

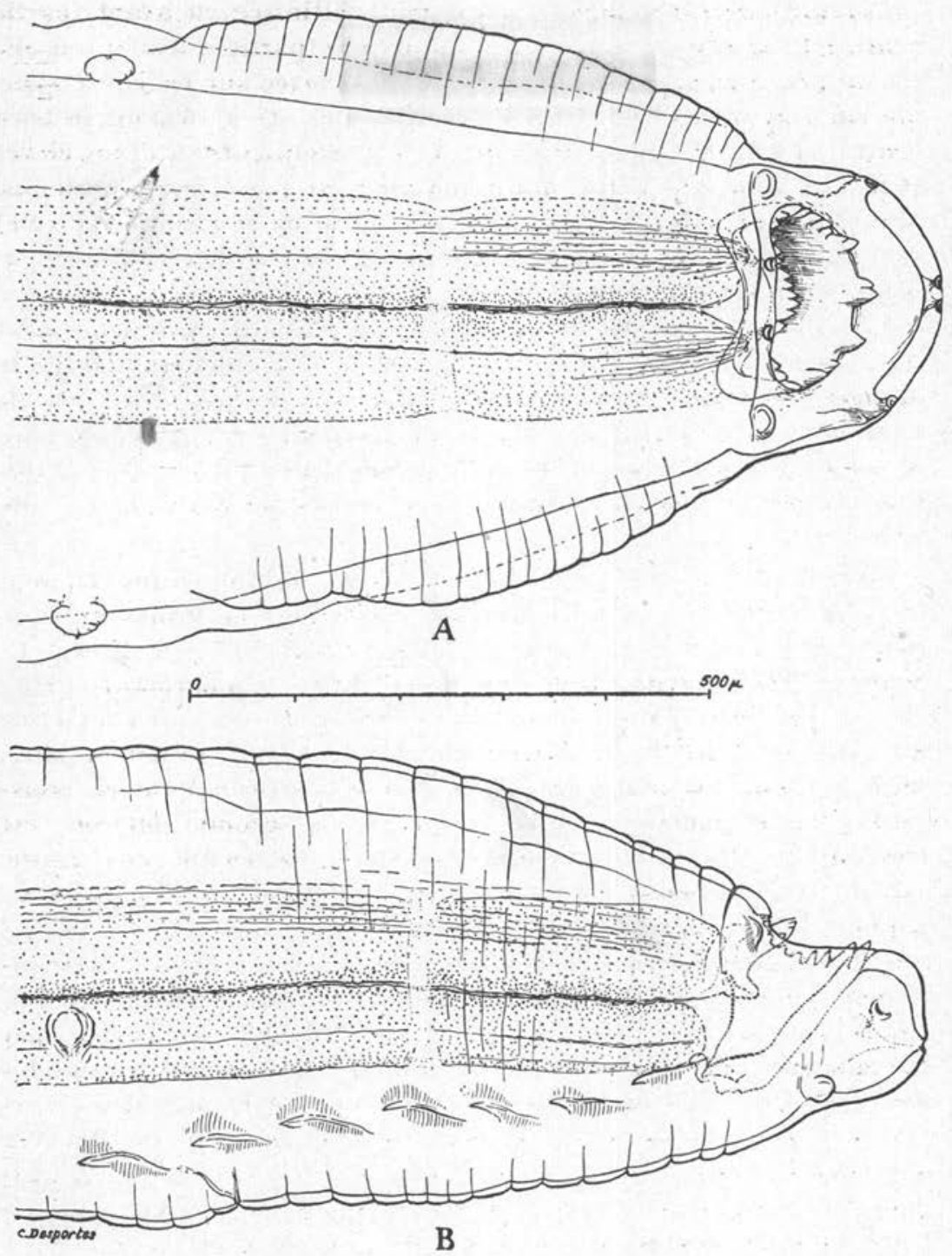

Fıg. 1. - Rictularia proni L. Seurat 1915 , femelle de $50 \mathrm{~mm}$., récoltée chez Sciurus nulgaris L. - A, extrémité antérieure, vue dorsale ; B, même extrémité, vue latérale.

files subventrales, une droite et une gauche. Exception faite pour la première paire de ces épines qui est presque parallèle au corps, 
toutes sont dirigées obliquement d'avant en arrière et de bas en haut; leur pointe se recourbe cependant légèrement vers la partie ventrale (ceci s'observe particulièrement sur les épines postérieures, en forme de griffe).

Les femelles à notre disposition présentaient 32 ou 33 paires d'épines prévulvaires (longues de 90 à $95 \mu$ ), quelquefoils ine paire au niveau de la vulve (de $65 \mu$ de longueur) et de 10 à 12 paires post-

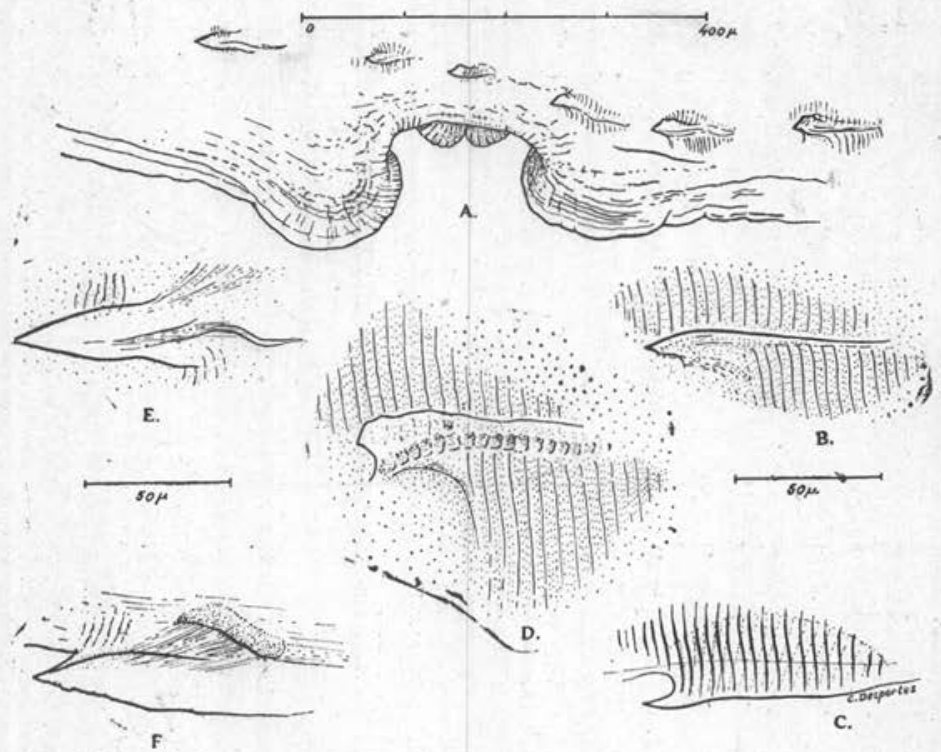

F1G. 2. - Rictularia proni L. Seurat 1915, femelle récoltée chez Sciurus vulgaris L. - A, région vulvaire, vue latérale. - B à F, épines cuticulaires du côté droit : $\mathrm{B}$, troisième épine, vue de face ; $\mathrm{C}$, cinquième épine, vue de profil ; $\mathrm{D}$, vingt-quatrième épine, vue de face ; $\mathrm{E}$, trente-sixième épine, vue de face ; F, la même, vue de profil.

vulvaires (de 70 à $80 \mu$ ), les dernières (situées à environ $8 \mathrm{~mm}$. de l'extrémité antérieure) étant très estompées. Toutes les épines prévulvaires présentent, en outre, une vingtaine de stries transversales, naissant de part et d'autre d'une petite crête située dans l'épaisseur de la cuticule, parallèle à l'axe de l'épine et du côté ventral, ce qui donne à ces épines l'aspect de peignes. Les stries s'estompent assez brutalement sur les épines post-vulvaires (fig. 2, E-F).

Dierides bien visibles, à 780 ou $820 \mu$ de l'extrémité antérieure, situées au-dessus de la ligne médio-latérale, au niveau de la septième paire d'épines ou un peu en avant. 
Champs latéraux relativement étroits, larges de $240 \mu$ dans la région vulvaire.

Pore excréteur situé au niveau de la sixième paire d'épines, à $707 \mu$ de l'extrémité antérieure chez une femelle de $48 \mathrm{~mm}$. 6 .

CEsophage long de $4 \mathrm{~mm}$. 65, large de $250 \mu$ en son milieu (femelle de $48 \mathrm{~mm} .6$ ) et progressivement élargi postérieurement, comprenant deux portions très peu distinctes, dont l'antérieure, un peu plus grêle $(210 \mu)$, mesure de $0 \mathrm{~mm}$. 93 à $1 \mathrm{~mm}$. 02 . Sa section est une étoile à trois branches dont les sommets, aigus, dessinent sur toute sa longueur trois lignes parallèles à la lumière centrale. L'œsophage se prolonge postérieurement par un intestin sensiblement de même diamètre et à peu près rectiligne.

Anneau nerveux peu visible, large de $18 \mu$ environ, situé, en son milieu, à $475 \mu$ de l'extrémité céphalique.

Vulve en avant de l'extrémité postérieure de l'œesophage chez les quatre femelles examinées (à $3 \mathrm{~mm}$. 96 de l'extrémité antérieure chez la femelle de $48 \mathrm{~mm}$. 6), formée de deux lèvres transversales très musculeuses, bordées elles-mêmes, extérieurement, d'un bourrelet souvent très épais et saillant. En arrière de la vulve se trouve le vagin, tube rectiligne, fortement musculeux, long de $1 \mathrm{~mm}$., et qui se bifurque postérieurement en deux branches utérines : ces dernières s'élargissent progressivement vers la partie postérieure du corps, qu'elles occupent presque entièrement, et remontent ensuite, en décrivant de nombreuses boucles, vers le tiers antérieur du corps où sont situés les ovaires pelotonnés (l'un d'eux étant légèrement postérieur à la jonction des utérus).

Anus représenté par une longue fente transversale, dont la lèvre antérieure recouvre la lèvre postérieure ; situé à $560 \mu$ de l'extrémité postérieure du corps.

Extrémité postérieure terminée par un appendice conique à la base duquel on observe, de chaque côté, l'orifice des phasmides.

Gufs petits, ovalaires $(50 \times 30 \mu$ environ $)$, à double coque épaisse $(2 \mu)$, transparente, laissant apercevoir un embryon enroulé.

\section{B. Individus récoltés chez Apodemus}

Sensiblement de même taille que les précédents, ils présentent des caractères identiques: la vulve est en avant de l'extrémité postérieure de l'œsophage ; 33 paires d'épines pectinées prévulvaires, souvent une paire au niveau de la vulve, 8 ou 9 ou 11 paires d'épines post-vulvaires. Même nombre de denticules aux mâchoires, même disposition et même forme des fausses dents œesophagiennes.

ANn. DE PaRASitologie, T. XX, Nos $1-2,1944-1945$. 
Discussion. - Nous avons comparé nos femelles de Richelieu à toutes les descriptions et figures que nous connaissions : de cette comparaison, il résulte qu'il s'agit de proni, sensu Seurat. Nos spécimens sont plus grands que ceux de Seurat, mais les proportions sont voisines., Même brièveté de l'œesophage (longueur approximativement égale au dixième de celle du corps) ; même emplacement des diérides, à peu de distance en arrière de l'anneau nerveux, au niveau de la septième paire d'épines pectinées; même nombre d'épines pectinées prévulvaires (33 d'après Seurat, 1916, p. 147), l'orifice vulvaire (en avant de la terminaison postérieure de l'œsophage) étant au niveau de la $34^{\circ}$ paire ; à peu près même nombre total de paires d'épines. Les dimensions des œufs sont à peu près correspondantes (Seurat donne $35-40 \times 25 \mu$ ). En ce qui concerne les papililes céphaliques, nous pensons que Seurat ne les a pas toutes observées, son matériel n'étant pas favorable : il en a signalé seulement quatre paires : nous en avons vu une paire supplémentaire. Dans la capsule buccale nous avons observé des denticules en plus grand nombre que n'en a figuré Seurat sur la « mâchoire » dorsale de $R$. proni, mais le nombre de ces productions cuticulaires n'est peut-être pas absolument constant; il est, en outre, parfois difficile à préciser.

La grosse dent triangulaire dorsale, décrite et figurée chez $R$. plagiostoma par Wedl et par Jägerskiöld (1909, p. 34 et pl. II, fig. 1, Z), est absente chez nos spécimens comme chez ceux de Seurat: nous n'avons trouvé qu'une seule dent épaisse et massive, la dent œsophagienne dorsale, figurée également par Seurat (1916, p. 148, fig. 1) et qui serait l'homologue de la dent $Z_{1}$ de $R$. plagiostoma, selon Jägerskiöld.

En comparant nos spécimens à la description et aux figures données par R.-Ed. Schulz pour amurensis, nous avons été frappés par la presque identité de la disposition des papilles céphaliques et des denticules de la capsule buccale : ceci nous permet de supposer qu'amurensis est extrêmement voisin de proni et peut-être seulement une variété de cette espèce; remarquons toutefois que si amurensis a aussi 33 paires d'épines prévulvaires, il en a un nombre (17) de post-vulvaires un peu plus élevé que nos spécimens et ceux de Seurat.

Nous sommes moins certains de l'identité spécifique des femelles rapportées à proni par E. Gendre (1921, p. 35-37) ; elles ont à peu près le même nombre total d'épines, pectinées ou non, qu'amurensis, mais la vulve est au niveau de la $42^{\circ}$ paire et les « papilles cervicales » (diérides) au niveau de la dixième paire. 
Gendre n'a malheureusement donné aucun renseignement sur les papilles céphaliques; il n'a pas non plus indiqué la position des denticules de la capsule buccale, laissant seulement présumer que l'ornementation de la bouche était conforme à celle décrite pour proni par Seurat.

\section{IV. - Hôtes ET Distribution GÉographiQue DES ESPÈcEs}

Par le tableau des hôtes et des lieux de récolte, nous voyons que les Rictularia sont répandus dans les régions chaudes et tempérées de l'Ancien et du Nouveau Continent et qu'ils peuvent se rencontrer chez des Mammifères de groupes très différents: Marsupiaux, Insectivores, Chéiroptères, Carnivores, Primates. En outre, il semble que, pour une partie, au moins, des espèces, il n'y a pas de spécificité parasitaire étroite. D'après les infestations constatées dans les jardins zoologiques, nous savons qu'une même espèce peut parasiter des hôtes de diverses origines, par exemple des Primates d'Amérique du Sud, d'Afrique, d'Asie, qui, dans la nature, ne sont évidemment pas hôtes normaux de la même espèce de Rictularia. Nous ignorons quel est l'hôte habituel et la patrie d'origine de $R$. alphi Lubimov, qui a si intensément (1) parasité huit espèces de Primates au jardin zoologique de Moscou.

Le fait que des Rictularia parasitent des Rats et des Souris et que ceux-ci peuvent être transportés à de grandes distances ainsi que des insectes hôtes intermédiaires, indique la possibilité de récolter une même espèce de Rictularia en des régions très éloignées de son aire naturelle de distribution.

Hôtes ET DISTRIBUTION GÉOGRAPHique DES ESPÈces DU GENRE Rictularia (2)

aethechini P.-L. Le Roux 1930.

in Ethechinus frontalis (A. Smith) : Insectivore.

Hamanskraal (Transvaal).

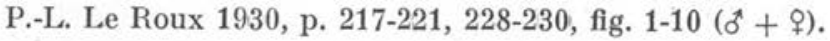

Sandground 1935, p. 160, 162 ; Lent et Freitas 1935 , p. 539.

affinis L.-A. Jägerskiöld 1904.

in Vulpes niloticus Desm. : Carnivore.

(1) Chez un seul Cebus fatuellus L., Lubimov (1933, p. 251) a compté 1.700 individus de $R$. alphi Lubimov.

(2) Les références en petits caractères sont celles où l'espèce est mentionnée, discutée ou comparée, sans indication nouvelle de caractères, d'hôte ou de lieu de récolte. 
Environs du Caire (Egypte).

L.-A. Jägerskiöld 1904, p. 660 ; 1909, p. 5, 6, 11, 16, 18-21, 25, $29,31,34,42,43,44$, pl. II, fig. 4, 6, $9\left(\sigma^{4}+\right.$ + $)$.

P. Sonsino 1888 , p. $115-119$ et 1888 , p. 192-196 (sous le nom de plagistoma $)\left(\sigma^{A}+\right.$ o $)$.

in Canis anthus Fr. Cuvier : Carnivore.

Tigzirt (Algérie).

et in Vulpes vulpes atlantica Wagner : Carnivore.

Bou Saâda (Algérie).

L. Seurat 1915 , p. $318-320$, fig. 3 ; 1917, p. $24(\overbrace{}^{+}+$o $)$.

in Canis (Thos.) adustus Sundevall : Carnivore.

Mt. Silinda (Rhodésie du Sud).

Sandground 1933, p. 292 (\%).

H. A. Baylis 1934 , p. 351 ; M. C. Hall 1913, p. 81-82 ; R. Hoeppli 1929, p. 76 ; H. F. Hsü 1935 , p. 595 : Lent et Freitas 1915 , p. 539 ; Lubimov 1933 , p. 259 ; Massino 1923 , p. 11 ; Seurat 1916, p. 149 ; Sandground 1935, p. 161,163 .

alphi M.-P. Lubimov 1933.

in Cebus apella L.,

Cebus fatuellus L.,

Cebus hypoleucus Humboldt,

Hapale jacchus L.,

H. (Leontocebus) rosalia L. = Mystax rosalia (L.),

Cercopithecus (Myopithecus) talapoin Erxleben,

Cercopithecus ruber $\mathrm{Gmel}$ = Erythrocebus patas $($ Schreb.),

Macaca rhesus (Audebert) $=M$. mulatta (Zim.) : Primates.

Jardin Zoologique de Moscou.

Lubimov 1933, p. 253-258, 251, fig. 1-8 (ô + +).

Lent et Freitas 1935, p. 540.

amurensis R.-Ed. Schulz 1927.

in « Souris » (gen. ? sp. ?) : Rongeur.

Troïtskoïé-sur-Toumel (district de Primorskaïa, U.R.S.S.).

R.-Ed. Schulz 1927, p. 61-63, fig. 17-20.

Lubimov 1933, p. 259 ; Lent et Freitas 1935, p. 539 ; Sandground 1935 , p. 160,162 .

bovieri R. Blanchard 1886.

in Vespertilio murinus Schreb. : Chẻiroptère.

Sarreguemines (Moselle).

R. Blanchard 1886, p. 297-304, pl. X, fig. 4-9 (q juv.).

Jägerskiöld 1909 , p. 39 ; Hoeppli 1929, p. 76 ; Lent et Freitas 1935, p. 539 ; Lubimov 1933 . p. 258 ; C. Parona 1889 , p. $774-775$; 1898 , p. 117.

cahirensis Jägerskiöld 1904.

in Felis catus domesticus : Carnivore.

Le Caire (Egypte). 
Jägerskiöld 1904 , p. $660 ; 1909$, p. 2-17, 18, 19, 20, 21, 25, 29, $30,34,42,43,44$, fig. texte I-VI, pl. I, fig. 3-5, pl. II, fig. 5, 7, $10\left(\sigma^{7}+q\right)$.

Abdel Azim 1939, p. 35 (jamais chez le chien).

Merv (Turkestan russe).

Massino 1923 , p. 3, 4, 9-14, fig. $3\left(\delta^{*}+q\right)$.

Merv (Turkestan russe) et Djeulfa (Arménie russe).

K.-I. Skrjabin et N.-P. Popov 1924, p. 60.

in Felis catus dom. et Canis familiaris L. : Carnivores.

Turkestan russe méridional.

A.-M. Petrov 1926, p. 6, 13, 14.

Arménie russe.

N.-P. Popov 1924, p. 5, 6, 7.

Vieille-Boukhara (Turkestan).

B. Massino 1925 , p. $67-69$, fig. 1 ; 1925 , p. $3,5(\delta+$ o 5 ,

Palestine.

G. Witenberg, 1934, p. 238.

in Canis familiaris L. : Carnivore.

Jérusalem.

G. Witenberg 1928, p. 603 (expériment.).

M. C. Hall 1913, p. 81-82 ; R. Hoeppli 1929 , p. 76 ; H. F. Hsü 1935 , p, 595 : Lent et Freitas 1935, p. 539 ; Lubimov 1@33, p. 260 ; A M. Petrov 1926, p. 13 ; Seurat 1916, p. 149 ; K. Sprehn 1930, p. 246. 258.

caucasica R.-Ed. Schulz 1927.

in Gerbillus meridianus Pallas : Rongeur.

Terekli-Mekteb (district de Kizlar, Caucase du Nord).

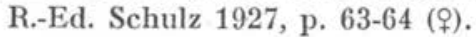

Lent et Freitas 1935 , p. 539 ; Lubimov 1933, p. 260 ; Sandground 1935, p. 162 .

citelli Mac Leod 1933.

in Citellus franklini (Sabine),

Citellus tridecemlineatus (Mitchill) $=$ Sciurus (Ictidomys) tridecemlineatus Mitchill: Rongeurs.

Manitoba (Canada).

J.-A. Mac Leod 1933, p. 114-116, fig. 1-4 $\left(\delta^{\circ}+q\right)$.

Lent et Freitas 1935, p. 540 ; Sandground 1935, p. 162, 164.

coloradensis M.-C. Hall 1916.

in Eutamias quadrivittatus (Say): Rongeur.

Pagosa Springs (Colorado).

M.-C. Hall 1916, p. 169, 173-175, 225, fig. 224-228.

in Peromyscns leucopus lencopus (Rafinesque) : Rongeur.

North Carolina.

R. Harkema 1936, p. 158, 164, 192.

A. B. Erickson 1938, p. 585 ; B. G. Chitwood et M. B. Chitwood 1937, p. 22, 29, fig. $23 \mathrm{LL}, 23 \mathrm{MMI}$; 1938, p. 62, 63: fig. $58 \mathrm{U}$; Goodrich 1932, p. 216 ; R. Hoeppli 1929, p. 76 ; I.ent et Freitas 1935 , p. 539 ; Lubimov 1933. p. 259 ; Sandground 1935 , p. $163,164$. 
cristata Frœlich 1802.

in Muscardinus avellanarius (L.) = Myoxus muscardinus Schreber : Rongeur.

Allemagne.

J.-A. Frœlich 1802, p. 9-13, 88, pl. I, fig. 1-3 (ㅇ).

in Apodemus sylvaticus (L.) : Rongeur.

Rennes (Ille-et-Vilaine).

F. Dujardin 1845 , p. 280-281 (q).

in Eliomys quercinus (L.) = Myoxus dryas Schreb., Glis glis (L.) : Rongeurs.

Musée de Vienne.

C.-A. Rudolphi, p. 61, 304 ; Diesing 1851, p. 245, 518.

in Apodemus sylvaticus (L.) et Mus musculus abbotti Waterhouse : Rongeurs.

Environs de Tiflis (Caucase).

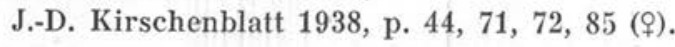

R. Blanchard 1886 , p. 301-303 ; Goodrich 1932, p. 218 ; M. C. Hall 1916, p. 169-170, fig. 216-217 ; Lent et Freitas 1935, p. 539 ; R. Hóeppli 1929, p. 76 : L. A. Jägerskiöld 1909, p. 38 ; Lubimov 1933, p. 258 ; C. Parona 1889 , p. 774,775 ; 1898 , p. 117 ; Sandground 1935 , p. 164 ; Seurat 1916 , p. 147 ; P. Sonsino 1888 , p. 194.

elegans Travassos 1928.

in Eumops perotis (Wied.) : Chéiroptère.

Engenheiro Gomide (S. Paulo, Brésil).

L. Travassos 1928 , p. 130-134, fig. 1-6 $\left(\sigma^{*}+q\right)$.

Lent et Freitas 1935, p. 539 ; Lubimov 1933, p. 259 ; Sandground 1235 , p. 162 .

elvirae C. Parona 1889.

in Sciurus (Dremomys) rufigenis Blanford : Rongeur.

Mt Mouleyit (Tenasserim, Birmanie).

C. Parona 1889 , p. 771-775, 780, pl. III, fig. $12-15$; 1898 , p. 117 ( $($ ).

H. A. Baylis 1928, p. 611 ; Hall 1916, p. 169, 170-171, fig. 218-221 ; R. Hoeppli 1929, p. 76 ; Jägerskiöld 1909, p. 39, 42 ; Lent et Freitas 1935, p. 539 ; Lubimov 1933, p. 258.

fallax L.-A. Jägerskiöld 1909.

in Sciurus melanogaster Thomas : Rongeur.

Ile Mentawei (Sud de Sumatra).

C. Parona 1898, p. 117 (sous le nom de plagiostoma) ; L.-A. Jägerskiöld 1909, p. 2, 35-38, 42, 43, fig. XIV, XV (९).

H. A. Baylis 1928, p. 611 ; Hall 1916, p. 169, 170-171, fig. 222-223 ; Hoeppli 1929 , p. 77 ; Lubimov 1933 , p. 260 ; Lent et Freitas 1935, p. 539 ; R. Ed. Schulz 1927, p. 64 ; Seurat 1915 , p. 322 ; 1916, p. 147 .

halli J.-H. Sandground 1935.

in Eutamias striatus lysteri Richardson : Rongeur. 
Douglas Lake (Michigan).

J.-H. Sandground 1935, p. 159-166, pl. XXVIII, fig. 5-9 (o+ + १).

Lent et Freitas 1935, p. 540.

harrisi H.-A. Baylis 1934.

in Mastomys coucha microdon (Peters) : Rongeur.

Morogoro (Tanganyika).

H.-A. Baylis, p. 349-352, fig. $6(\widehat{o}+$ +).

H. T. Chen 1936, p. 283-284; Lent et Freitas 1935, p. 540.

houdemeri H.-F. Hsü 1935.

in Viverra zibetha L. : Carnivore.

Langson (Tonkin).

H.-F. Hsü 1935, p. 592-595, fig. 24-30 $\left({ }^{*}+q\right)$.

Lent et Freitas 1935, p. 540 ; Tubangui et Masilungan 1938, p. 263.

jaegerskioeldi H. Lent et T. de Freitas 1935.

in Caluromys philander (L.) : Marsupial.

Tijuca (Rio de Janeiro, Brésil).

H. Lent et J.-F. Teixeira de Freitas, 1935, p. 535-539, fig. 1-8 ( $\left.\delta^{7}\right)$.

macdonaldi (G.-E. Dobson 1880).

in Megaderma frons E. Geof. St-Hil. : Chéiropière.

Goldcoast.

G.-E. Dobson 1880, p. 412-414 (dans le genre Pterygodermatites) ; 1880, p. 583 (sans nom) ; J.-D. Mac Donald 1880, p. 409-411, pl, XXI, fig. 1-9.

Nil Blanc.

L.-A. Jägerskiöld 1909, p. 2, 5, 7, 8, 11, 15, 20, 21, 30, 34, 42, 43, fig. VII-X, pl. I, fig. 1, 2, 6, pl. II, fig. 2, 3, 8 ( $\sigma^{+}+$+ $)$.

R. Hoeppli 1929, p. 76 ; Jägerskiöld 1904, p. 660 ; Lent et Freitas 1935. p. 539 ; Lubimov 1933 , p. 259 ; C. Parona 1889 , p. 775 ; Sandground 1935. p. 160,163 : L. Seurat 1915 , p. 322 ; 1916, p. 147 ; 1917, p. 24.

macdonaldi (Dobson), sensu L. Seurat 1915.

in Genetta bonapartei Loche : Carnivore.

Alger.

L.-G. Seurat 1915 , p. 320 ; 1917 , p. 24 (o').

magna H.-A. Kreis 1937.

in Mus sp.: Rongeur.

? (Collection O. Fuhrmann).

H.-A. Kreis 1937 , p. 495-496, fig. A-E (o).

mjöbergi H.-A. Baylis 1928.

in Arctitis binturong Rafll. : Carnivore.

Mt Penrissen (Sarawak).

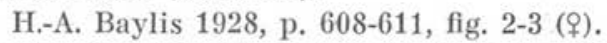

in Paradoxurus (Paguma) larvatus larvatus Temminck: Carnivore. 
Kwantung Province (Chine).

H.-T. Chen 1937, p. 419-422, fig. 1-6 $(\hat{o}+\uparrow)$.

Lent et Freitas 1935, p. 539 ; Lubimov 1933, p. 260 ; Hsü 1935, p. 595 ; Sandground 1933 , p. $575 ; 1935$, p. $160,162$.

muris B. Galli-Valerio 1932.

in Mus musculus L. : Rongeur.

Lausanne (Vaud, Suisse).

B. Galli-Valerio 1932, p. $137\left({ }^{\star}\right)$.

Lent et Freitas 1935, p. 539 ; Sandground 1935, p. 162.

paradoxuri M.-A. Tubangui et V.-A. Masiluñgan 1938.

in Paradoxurus philippinensis Jourdan : Carnivore.

Balanga (Luzon, Philippines).

M.-A. Tubangui et V.-A. Masiluñgan 1938, p. 262-263, 267, pl. III, fig. 1-3 $(\hat{\sigma}+\uparrow)$.

plagiostoma (C. Wedl 1861) (nec Willemoes-Suhm 1873 sensu).

in Erinaceus auritus E. Geof. St-Hil. = E. libycus Ehrenb. : Insectivore.

Egypte.

C. Wedl 1861, p. 464-466, 481, pl. I, fig. 5-11 (in gen. Pterygodermatites $)(\hat{\sigma}+q)$.

Alexandrie (Egypte).

L.-A. Jägerskiöld 1909, p. 2, 18, 30, 31-35, 37, 38, 42, 43, fig. 11-

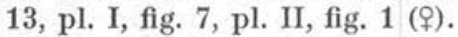

R. Blanchard 1886, p. 302-303 ; M. C. Hall 1916, p. 171, 172 ; Hoeppll 1929 , p. 76 ; Jägerskiöld 1904 , p. 660 ; Lent et Freitas 1935 , p. 539 ; Lublmov 1933, p. 259 ; C. Parona 1889 , p. 774-775 ; 1898, p. 117.: P. Sonsino, p. $115-119$; 1888 , p. $192-196$; Seurat 1915 , p. 322 ; 1916 , p. 147, 149 ; Sandground 1935 , p. 160.

plagiostoma (C. Wedl 1861) sensu R.-T. Leiper 1911.

in Paradoxurus (Paguma) larvatus Tem. : Carnivore.

Jardin Zoologique de Londres.

R.-T. Leiper 1911, p. 620-621 (sans description).

proni L.-G. Seurat 1915.

in Herpestes ichneumon L. : Carnivore.

Bordj-Menaïel (Kabylie, Algérie).

L.-G. Seurat 1915, p. 320-322, fig. 1-2 (q).

in Herpestes ichneumon L. : Carn. + Arvicanthis barbarus (L.) :

Rongeur.

Bordj-Menaïel.

L.-G. Seurat 1916, p. 146-149, fig. 1-2 ; 1917, p. 24 ; 1930, pl. XVI, fig. 1-2 $\left(\sigma^{\circ}+\right.$ क $)$.

M. C. Hall 1916 , p. 169,225 ; R. Hoeppli 1929 , p. 76 ; H. F. Hsü 1935 , p. 595 ; H. A. Kreis 1937 , p. 496 ; Lent et Freitas 1935 , p. 539 ; Lubimov 1933, p. 259 ; Sandground 1935, p. 164 ; R. E. Schulz 1927, p. 63. 
proni' L.-G. Seurat 1915, sensu E. Gendre 1921.

in Gerbillus emini Thomas : Rongeur.

Abomey (Dahomey).

E. Gendre 1921 , p. 35-37, fig. 6 ; 1928, p. 73 (q).

Réf. ef. proni Seurat 1915.

scalopis A.-L. Goodrich 1932.

in Scalops aquaticus (L.) : Insectivore.

Manhattan (Kansas).

A.-L. Goodrich 1932, p. 216-218, pl. XXX, fig. 1-6 (†).

Lent et Freitas 1935, p. 539 ; Sandground 1935, p. 162 (scapopis).

spinosa (R. v. Willemoes-Suhm 1869).

in Vesperugo mystacinus Leisler: Chéiroptère.

Berchtesgaden (Bavière).

R. v. Willemoes-Suhm 1869, p. 473-474 (in gen. Ophiostomum);

1873, p. 331-332, pl. XVII, fig. 1 (sous le nom de Rictularia plagiostoma) (官.

Linstow 1909, p. 67-68, fig. 49 ; Jägerskiöld 1909, p. 31, note ; P. Sonsino 1888 , p. 115 ; 1888 , p. 192.

splendida M.-C. Hall 1913.

in Canis nebracensis Merriam : Carnivore.

Amo (Colorado).

M.-C. Hall 1912 (Rictularia sp.); 1913, p. 73-83, fig. 1-6 (రో + १).

R. Hoeppli 1929, p. 76 ; H. F. Hsü 1935, p. 595 ; A. L. Goodrich, 1932, p. 216 ; Lent et Freitas 1935 , p. 539 ; Le Roux 1930, p. 218 ; Lubimov 1933 , p. 260 ; Massino 1923, p. 11 ; Sandground 1935, p. 162 ; Seurat 1915 , p. 320 : G. Steiner 1919 , p. 39,96 , pl. III, flg. 12.

tani Hoeppli 1929.

in Mus norvegicus Erxleben : Rongeur.

Amoy (Chine).

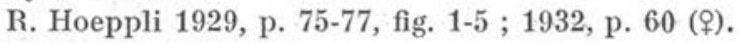

Mus rattus L. : Rongeur.

Canton (Chine).

H.-T. Chen 1933, p. 68, 70, 71, 74 (Rictularia sp.) (ઠో).

Mus norvegicus Erxl. + Mus rattus L. : Rongeurs.

Canton (Chine).

H.-T. Chen 1936, p. 283-286, fig. $1\left(\sigma^{*}+q\right)$.

H. A. Baylis 1934, p. 352 ; R. Hoeppli 1929, p. 77 ; Lent et Freitas 1935, p. 539 ; Lubimov 1933 , p. 259 ; Sandground 1935 , p. 160, 162 ; Tubangui 1931, p. 585 .

taterIIIi H.-A. Baylis 1928.

in Taterillus gracilis angelus Thomas et Hinton : Rongeur.

Kano (Nigeria).

H.-A. Baylis 1928, p. 301-302, fig. 22-24 (ó + क).

H. A. Baylis 1934, p. 349, 351, 352 ; H. T. Chen 1936, p. 283-284 ; Lent et Freitas-1935, p. 539 ; Lubimov 1933, p. 259 ; Sandground 1935, p. 162. 
vulpis Galli-Valerio 1932.

in Vulpes vulpes L. : Carnivore.

Fully (Vaud, Suisse).

B. Galli-Valerio 1932 , p. $135\left(\sigma^{7}\right)$.

whartoni M.-A. Tubangui 1931.

in Mus norvegicus Erxleben : Rongeur.

Manila (Philippines).

M.-A. Tubangui 1931 , p. 579-581, fig. $18 a-18 c$ (१).

H. A. Baylis 1934 , p. 352 ; H. T. Chen 1933. p. 68 ; 1936, p. 285 ; Lent et Freitas 1935 , p. 539 ; Sandground 1935 , p. 162.

wheeleri Sandground 1933.

in Paradoxurus (Paguma) larvatus intrudens (Wroughton): Carnivore.

Lai Chau (Tonkin).

J.-H. Sandground 1933, p. 573-575 (q).

H. T. Chen 1937 , p. 422 ; H. F. Hsü 1935 , p. 595 ; Lent et Freitas 1935 , p. 540 ; Sandground 1935, p. 162.

sp.

in Ethosciurus byatti (Kershaw) : Rongeur.

Morogoro (Tanganyika).

H.-A. Baylis 1934, p. 352 (官).

Lent et Freitas 1935, p. 540.

sp.

in Potos flavus (Schreber) = Cercoleptes caudivolvulus (Pallas):

Carnivore.

Parc Zoologique de New-York.

G.-W. Mac Clure 1934 , p. 51, 58 (官.

Lent et Freitas 1935, p. 540.

sp.

in « Rat»: Rongeur.

Tonkin.

B. Schwartz 1925 , p. 7 (sans description).

sp.

in Macroscelides rozeti : Insectivore.

Djebel-Santon (Algérie).

E. Brumpt, 3-8-1899. Manuscrit (q).

\section{V. - ESPÈCES QUI ONT ÉTÉ RETIRÉES DU GENRE Rictularia}

1. Riciularioides amphiacantha (Dies. 1851) M.-C. Hall 1916. Cette espèce est connue seulement par la femelle, trouvée dans l'intestin grêle d'un Rongeur : Oxymycterus rufus (Desm.) = Lemmus dasytrichus (Wied.) [Muridæ] à Ytararé (Brésil) ; a été décrite sous le nom d'Ophiostomum amphiacanthum Diesing (1851, p. 246 ; 1857, 
p. 16-17, 25, pl. II, fig. 29-36), puis placée par Diesing lui-même (1861, p. 648) dans le genre Rictularia. Les trois spécimens-types ont été revus par Richard von Drasche (1883, p. 123, 136, pl. VII, fig. 23, deux épines cuticulaires) qui laissa l'espèce dans le genre Rictularia Parona (1898, p. 117) rappela qu'amphiacantha a trois files d'épines. Jägerskiöld $(1909$, p. 40) estima que si la description et les figures données par Diesing étaient exactes, il faudrait établir un genre nouveau. Ce genre, Rictularioides, fut proposé par M.-C. Hall (1916, p. 168, 175-176, fig. 229-233), qui a reproduit les figures publiées par Diesing en 1857. A notre connaissance, l'espèce n'a pas été retrouvée.

2. Rictularia disparilis V. Irwin Smith (1922, p. 311-317, fig. 1-6). - Cette espèce a été décrite d'après deux femelles trouvées par J. Burton Cleland dans le tube digestif d'un Lézard qui, d'après Irwin Smith (1922, p. 427), est Lygosoma (Liolepisma) entrecasteauxi Duméril et Bibron 1839 (Scincidæ) des îles Flinders (Australie).

La bouche est une fente dorso-ventrale à l'extrémité de l'axe longitudinal du corps (elle ne se trouve pas déplacée à la face dorsale, comme chez Rictularia), entre deux lèvres latérales asymétriques ; la droite, un peu plus haute que la gauche, porte une dent médiane conique semblable à la dent labiale externe des Physaloptera; la gauche porte une série de trois lobes, ou plus, aigus, denticulés à leur sommet. Appliquée extérieurement à chaque lèvre, se trouve une épaisse protubérance hémisphérique, pourvue de deux grandes papilles. A la base des lèvres, la tête est entourée d'un étroit collier cuticulaire, circulaire, à bord libre ; un peu en arrière de cette collerette, la tête forme un renflement circulaire, délimité par un profond sillon. A partir de la papille post-cervicale gauche, tout le long du côté gauche du corps, il y a une crête régulièrement sinueuse, dont chaque sinuosité porte une épine dirigée postérieurement; ces épines se trouvent portées alternativement par le côté droit et le côté gauche de la crête cuticulaire ; la plus antérieure correspond au niveau de la jonction de l'œsophage musculaire avec l'œsophage glandulaire. Du côté droit, de même que sur les faces dorsale et ventrale, il n'y a pas trace de crête ou d'épines. Sur la crête parcourant le côté gauche, on compte 108 épines, soit 54 sur chacun des côtés de celle-ci. La vulve est située ventralement vers la mi-longueur de l'œsophage, vers le niveau de la $13^{\circ}$ épine. L'organisation interne est celle des Physalopterinz.

Irwin Smith a mis en évidence les rapports étroits de disparilis avec Physaloptera, mais a cependant estimé qu'il s'agissait d'un Rictularia, disant que, d'après Frolich et Dujardin, le type du 
genre, cristata, ne possède qu'une seule file d'épines, contrairement à toutes les autres espèces placées dans le genre Rictularia; il faudrait ainsi, selon Irwin Smith, si cristata n'a bien qu'une seule file d'épines, établir un nouveau genre pour toutes les espèces considérées par Jägerskiöld et Hall comme des Rictularia, ne laisser dans Rictularia que cristata et disparilis et faire passer ce genre dans la sous-famille des Physalopterinæ L. Seurat 1913.

En réalité, le texte de Frœlich et celui de Dujardin nous semblent pouvoir être autrement interprétés que par Irwin Smith(1): Frœlich dit que le cou est armé " auf der einen Seite mit einer ununterbrochenen Reihe von Sägezähnchen », l'on peut comprendre que, pour chaque côté, il y a une file ininterrompue de denticules pectinés, et non pas, comme le fait croire la figure 3 de Frœlich, d'un côté seulement ; Dujardin dit que le tégument montre " deux bandes longitudinales internes... portant, à partir de la tête jusqu'à la vulve, une rangée non symétrique de 18 à 20 crochets oblongs et obliques », cela veut dire que chacune des bandes longitudinales -- et non une seule - porte une file longitudinale de crochets; Dujardin a donc bien vu deux files longitudinales d'épines pectinées (2). Il est évident que disparilis n'appartient pas au genre Rictularia, comme l'a déjà fait remarquer R. Hoeppli (1929, p. 76). Nous proposons donc pour l'espèce d'Irwin Smith un genre Pseudorictularia, dont la diagnose sera provisoirement celle donnée pour l'espèce-type : Pseudorictularia disparilis (v. Irwin Smith, 1922) et que nous placerons en appendice aux Physalopterinæ.

3. Rictularia paradoxa O. von Linstow (1903, p. 272-273, pl. XVIII, fig. 5). - Cette espèce, décrite d'après une femelle d'origine inconnue du Musée de St-Pétersbourg, fut reconnue identique à Gnathostoma shipleyi Stossich (1900, p. 1-2, pl. I, fig. 1-5) de Diomedea exulans L. du Pacifique-Ouest, ainsi que l'a admis Linstow luimême (1904, p. 301). Jägerskiöld (1909, p. 40) précisa que ce n'était pas un Rictularia, néanmoins c'est sous le nom de Rictularia shipleyi (Stoss.) que nous trouvons cette espèce signalée par T.-H. Johnston (1912, p. 106) et par J.-B. Cleland (1922, p. 89) chez Daption capensis (L.) (Procellariiforme) des environs de Sydney.

K.-I. Skrjabin (1916, p. 972) a reconnu que la même espèce avait été décrite sous le nom de Acuaria pelagica Seurat (1916, p. 785-787, fig. 1-5) du ventricule succenturié de Larus canus L. à Mers-el-Kébir

(1) Voir l'interprétation de M. C. Hall (1913, p. 76-77).

(2) Dujardin n'a pas vu ces deux files symétriques l'une par rapport à l'autre. Peut-être parce que les épines pectinées, étant mobiles sur le vivant, peuvent ainsi prendre un aspect différent d'un côté et de l'autre, donnant une impression d'asymétrie. 
et de Puffinus kuhli (Boie) à Alger. C'est le type du genre Seuratia K.-I. Skrjabin (1916, p. 973, diagnose) [Fam. Acuariidæ Seurat 1913, s.f. Seuratiinæ B.-G. Chitwood et E.-E. Wehr 1932] (1). L'appellation Seuratia shipleyi (Stossich, 1900) K.-I. Skrjabin 1916 paraît définitivement acceptée ; elle a été adoptée par L.-G. Seurat (1930, p. 560, fig. 25).

\section{RÉSUMÉ}

1. Nous exposons quelle est actuellement la position de Rictularia dans le système des Spiruroidea et quels sont les caractères utilisés pour la discrimination des espèces. Nous donnons un tableau indiquant pour la femelle de chaque espèce le nombre de paires d'épines pectinćes, ainsi que le niveau de la vulve par rapport à ces formations.

2. Nous attirons l'attention sur l'insuffisance de nos connaissances sur le type du genre, $R$. cristata Frolich 1802, et nous en concluons que, tant qu'il n'aura pas été redécrit, il restera, au point de vue de sa morphologie, une espèce inquirenda.

3. Nous décrivons les spécimens trouvés par nous à Richelieu (Indre-et-Loire) : cinq femelles chez un Apodemus sylvaticus (L.) et quatre femelles chez deux Sciurus vulgaris L. ; nous les identifions à $R$. proni Seurat 1915, décrit par Seurat d'après des individus trouvés en Algérie, une fois chez une Genette et plusieurs fois chez le Rat rayé. C'est la première fois que proni est signalé en France et en Europe. Nous estimons comme très voisin R. amurensis Schulz 1927, trouvé chez une Souris indéterminée en Russie.

4. Nous donnons un tableau des hôtes et de la distribution géographique pour toutes les espèces connues de Rictularia, avec références bibliographiques.

5 . Nous passons rapidement en revue les espèces qui ont été attribuées à tort au genre Rictularia et, pour $R$. disparilis Irwin Smith 1922, nous proposons le genre Pseudorictularia.

(1) Ne pas confondre avec la s.f. Seuratinæ Hall 1916 (=Seuratidæ A. Railliet) créée pour Seuratum Hall 1916 et actuellement placée dans la famille des Cucullanide Th.-Sp. Cobbold 1864.

P.S. - Nous avons eu connaissance trop tardivement de la description de Rictularia onychomis Ashton C. Cuckler (Journ. Parasitology, XXV, n. 5, oct. 1939 , p. 431-435, fig. 1-6) pour en tenir compte dans notre travail.

Cette espèce a été trouvée dans la paroi de l'intestin d'Onychomys leacogaster (Wied) [Rodentia] près de Valentine (Nebraska) et décrite d’après trois femelles longues de 24 à $42 \mathrm{~mm}$.; il y a 56-60 paires de processus cuticulaires (26-27 paires de peignes et 30-33 paires d'épines) dont $32-33$ prévulvaires. La vulve est antérieure à l'extrémité postérieure de l'œsophage. La bouche est terminale. Les œufs mesurent env, $45 \times 33 \mu$. A.-C. Cucikler a donné une clef pour la détermination des femelles du genre. 


\section{BibLIOGRAPHIE}

1939, Azıм (M.-A.). - Helminthes parasites des chiens et des chats en Egypte. Annales de Parasitologie, XVII, n ${ }^{\circ}$, janv. 1939, p. 32-36.

1928, Bayiss (H.-A.). - Some further Parasitic Worms from Sarawak. Annals and Mag. Natur. History, Sér. 10, I, may 1928, p. 606-615, fig. 1-3.

1928, - On a collection of Nematodes from Nigerian Mammals (chiefly Rodents). Parasitology, XX, $\mathrm{n}^{\circ} 3$, 1928, p. 280-304, fig. 1-25.

1934, - On a Collection of Cestodes and Nematodes from small Mammals in Tanganyika Territory. Annals and Mag. Natur. History, Sér. 10, XIII, 1934, p. 338-353, fig. 1-6.

1886, Blanchand (R.). - Notices helminthologiques (Première série). Bull. Soc. Zool. de France, XI, fasc. 1-3 ; 18-3-1886, p. 294-304, pl. X, fig. 1-9.

1933, CHEN (H.-T.). - A preliminary report on a survey of animal parasites of Canton, China, Rats. Lingnan Science Journal, XII, $\mathrm{n}^{\circ} 1$, febr. 2, 1933 , p. 65-74.

1936, - Rictularia tani Hoeppli from Rats in Canton (Nematoda : Rictulariidx). Lingnan Sc. Journ., XV, $\mathrm{n}^{\circ} 2$, june 20, 1936, p. 283-286, fig. 1.

1937, - Some parasitic Nematodes from Mammals of South China. Parasitology, XXIX, $\mathrm{n}^{\circ} 4,1-10-1937$, p. 419-434, fig. 1-22.

1934, Chrtwoon (B.-G.) et Werr (E.-E.). - The value of cephalic structures as characters in Nematode classification, with special reference to the superfamily Spiruroidea. Zeitschrift für Parasitenkunde, VII, Heft 3, 19-12-1934, p. 273-335, fig. texte 1-20, pl. I.

1922, Creland (J.-B.). - The parasites of Australian Birds. Transact. Royal Soc. of South Australia, XLVI, 1922, p. 85-118.

1851, Diesing (C.-M.). - Systema Helminthum, II, Vindobonæ. 1851 , vi + 588 p. + Corrigenda 3 p.

1857, - Sechzehn Arten von Nematoideen. Denkschriften mathem. naturwissensch. Classe der Kaiserl. Akad. der Wissenschaften, Wien, XIII, 1857 , p. $6-26$, pl. I-IV.

1861, - Revision der Nematoden. Sitzungsberichte d. Kaiserl. Akad. d. Wissenschaften. Mathem. Naturwissensch. Classe, Wien, XLII, 1861, $\mathrm{n}^{\circ} 28$, p. 595-736, pl.-fig. 1-11.

1883, Drasche (R. von), - Revision der in der Nematoden-Sammlung der K.K. zoologischen Hofcabinetes befindlichen Original-Exemplare Diesing's und Molin's. Verhandl. der K.K. zoolog. botan. Gesellschaft in Wien (Jahrg. 1882), XXXII, 1883, p. 117-138, pl. VII-X.

1880, Dobson (G.-E.). - An Annelidan Entozoon. Nature. London, XXII, n 573 , 21-10-1880, p. 583.

1880, - Note on Pterygodermatites Macdonaldii, the type of a new order of vermes. Annals and Mag. Natural History. London, 5 Sér., VI, $\mathrm{n}^{\circ} 36$, déc. 1880 , p. $412-414$.

1938, Erickson (A.-B.). - Parasites of some Minnesota Cricetide and Zapodide, and a Host Catalogue of Helminth Parasites of Native American Mice. American Midland Naturalist, XX, $\mathrm{n}^{\circ} 3$, nov. 1938, p. 575-589, fig. 1-18.

1802, Frcelich (J.-A. von). - Beiträge zur Naturgeschichte der Eingeweidewürmer. Naturforscher, Halle. Stück XXIX (1802), p. 5-96, pl, I, fig. 1-21, pl. II, fig. 1-25. 
1932, Galli-Valerio (B.). - Notes de parasitologie et de technique parasitologique. Zentralblalt für Bakteriol. Parasitenk. Abt. I. Orig., CXXV, Heft 1-2, 6-7-1932, p. 129-142, fig. 1-3.

1921, Gendre (F..). - Sur dèx espèces de Nématodes africains. Actes Soc. Linnéenne Bordeaux, LXXIII, séance du 5-1-1921, p. 28-36, fig. 1-6.

1928, - Nématodes (in Ch. Joyeux, E. Gendre, J.-B. Baer, Recherches sur les Helminthes de l'Afrique Occidentale française), p. 54-81, fig. 45-52. Société de Pathologie exotique. Monographie II, Paris, 1928.

1932, Goodrich (A.-L.). - Rictularia scalopis, sp. nov., a Nematode from the Mole (Scalops aquaticus (Linn.). Transact. American Microscopical Soc., LI, n 3 , july 1932, p. 216-218, pl. XXX, fig. 1-6.

1912, Halr. (M.-C.). - The Parasite Fauna of Colorado. Colorado College Publications. General Series, $\mathrm{n}^{\circ}$ 59-60 ; Science Series, XII, $\mathrm{n}^{\circ} 10$, jan.march 1912, p. 329-384.

1913, - A new Nematode, Rictularia splendida, from the Coyote, with notes on other Coyote parasites. Proceed. Un. St. Nat. Museum, $\mathrm{n}^{\circ}$ 2012, XLVI, 23-8-1913, p. 73-84, fig. 1-6.

1916, - Nematode parasites of Mammals of the orders Rodentia, Lagomorpha and Hyracoidea. Proceed. Un. St. Nat. Museum, n ${ }^{\circ}$ 2131, L, 13-51916, p. 1-258, fig. 1-290, pl. I. Errata 1 p.

1936, HaRkema (R.). - The parasites of some North Carolina Rodents. Ecological Monographs, VI, $\mathrm{n}^{\circ} 2$, april 1936, p. 151-232, fig. 1-5.

1929, Hoeppli (R.). - Rictularia tani n. sp., ein Parasit des Rattendarmes. Zentralbl. für Baliteriol. Parasitenkunde, I Abt., Orig., CX, Heft 1/3, 2-1-1929, p. 75-78, fig. 1-5.

1932, - Parasitic and free-living Nematodes found on the Island of Amoy, Marire Biol. Assoc. China. First Annual Report, 1932, p. 57-63.

1935, Hsü (H.-F.). - A Study of some Strongyloidea and Spiruroidea from French Indo-China and of Thelazia chungkingensis Hsü, 1933, from China. Zeitschrift für Parasitenkunde, VII, 5 Heft, 20-6-1935, p. 579600, fig. 1-31.

1922, Irwin Smith (V.). - A new Nematode Parasite of a Lizard. Proceed. Linnean Soc. New South Wales, XLVII, part 3, 15-9-1922, p. 311-318, fig. 1-17.

1922, - Notes on Nematodes of the genus Physaloptera. Part IV. The Physaloptera of Australian Lizards (continued). Proceed. Linnean Soc. New South Wales, XLVII, part 4, 15-12-1922, p. 415-427, fig. 1-38.

1904, Jafgerskioeld (L.-A.). - Ueber einige Rictularien aus Egypten und dem Sudan. (Naturvetenskapliga Studentsällskapet, Upsala. Sitzung den 6 nov. 1903). Zool. Anzeiger, XXVII, Nr. 20/21, 14-6-1904, p. 660.

1909, - Nematoden aus Egypten und dem Sudan (eingesammelt von der Schwedischen Zoologischen Expedition), I. Rictularia und Dichelyne. Results of the Swedish Zoological Expedition to Egypt and the White Nile 1901 under the Direction of L.-A. Jägerskiöld, Part III, No 25, Upsala, 1909, p. 1-66 + I-1v, fig. texte 1-23, pl. I-IV. 1912, Johnston (T.-H.). - Internal parasites recorded from Australian Birds. The Emu, XII, part II, 1-10-1912, p. 105-112.

1938, Kirschenblatt (J.-D.). - Die Gesetzmässigkeiten der Dynamik der Parasiten-fauna bei den Mäuseähnlichen Nagetieren (Muriden) in Transkaukasien. Edition de l'Université d'Etat de Leningrade. 1938. 92 pages, 22 figures. 
1937, KreIs (H.-A.). - Beiträge zur Kenntnis parasitischer Nematoden. IV. Neue und wenig bekannte parasitische Nematoden. Zentralbl. für Bakteriol. Parasitenkunde, I Abt., Orig., CXXXVIII, Heft 7/8, 31-3-1937, p. 487-500, fig. $1 \mathrm{~A}-6 \mathrm{E}$.

1911, Leiper (R.-T.). - Demonstration of Nematode Parasites obtained from animals in the Zoological gardens during the year ending November 1910. Proc. Zool. Soc., London, $\mathrm{n}^{\circ}$ XLII-XLiv, april 4, 1911, p. 620-621.

1935, Lent (H.) et Freitas (J.-F. Teixeira de). - Sobre dois novos nematodeos parasitos da quica: Caluromys philander (L.). Memor. Instit. Oswaldo Cruz, XXX, fase. 3, dez. 1935, p. 535-542, fig. 1-10.

1930, Le Rotx (P.-L.). - A new Nematode (Rictularia athechini sp. nov.) a Physaloptera and an Acanthocephala from the hedgehog (Ethechinus frontalis). XVIth Report Director Veterinary Services and Animal Industry, Union of South Africa. Pretoria, aug. 1930, p. 217232, fig. 1-18.

1903, Linstow (O. von). - Entozoa des zoologischen Museums der Kaiserlichen Akademie der Wissenschaften zu St-Petersburg. II. Annuaire Musée zoologique Acad. Impér. Sciences, St-Pétersbourg, VIII, nov. 1903, p. 265-294, pl. XVII-XIX, fig. 1-36.

1904, - Beobachtungen an Nematoden und Cestoden. Arch. für Naturgersch., Jahr. LXX, I, (3), aug. 1904, p. 297-309, pl. XIII, fig. 1-29.

1909, - Parasitische Nematoden. Süsswasserfauna Deutschlands. Jena, 1909. Heft 15 , p. $47-83$, fig. 1-80.

1933, Lubimov (M.-P.). - Rictularioseseuche bei Affen der Moskauer Tiergartens. Zeitschrift Infektionskrankheiten Haustiere. Berlin, XLIV, Nr. 4, 9-81933, p. 250-260, fig. 1-8.

Mac Clune (G.-W.). - Nematode Parasites of Mammals. From specimens collected in the New-York Zoological Park, 1932. Zoologica, NewYork, XV, No 3,1934 , p. 49-60.

1880, Macnonald (J.-D.). - On the Anatomy of a new Parasitic Worm found in the Intestine of a Bat (Megaderma frons). Annals and Magazine of Natur. History. London, 5 ser., VI, $\mathrm{N}^{\circ} 36$, dec. 1880 , p. 409-411, pl. XXI, fig. 1-9.

1933, Mac LeOd (J.-A.). - A parasitological Survey of the genus Citellus in Manitoba. Canadian Journal of Research, IX, aug. 1933, p. 108-127, fig. texte 1-18, pl. I, fig. 1-2.

1923, Massino (B.-G.). - Faune des Nématodes des Chats du Turkestan et comparaison avec celle de quelques régions de la Russie d'Europe. Supplément à: Vestnik Mikrobiologie $i$ Epidemiologie. Saratov. 1923, p. 1-16, fig. $1-3$.

1925, - Contribution à l'étude de la faune helminthologique de la VieilleBoukhara. (Travaux helminthologiques de la mission scientifique pour l'étude des maladies parasitaires en Boukhara, pendant la période du 20-5 au 30-8-1923). Journal Russe de Médecine Tropicale, $1925, \mathrm{n}^{\circ}, 4-5-6$. Tirage à part, 8 pages.

1925, - Ein neuer Nematode des Hundes : Rictularia cahirensis Jägerskiöld 1909. Berliner Tierärztliche Woch., XLI, 1925, Nr. 5, p. 67-69, fig. 1.

1889, Parona (C.). - Sopra alcuni elminti di Vertebrati Birmani raccolti da Leonardo Fea. Annali del Museo Civico di Storia Naturale di Geno$v a$, ser. $2^{\circ}$, VII (XXVII, 1889), 10-14 Ottobre 1890, p. 765-780, pl. III, fig. 1-18. 
1859, Parona (C.). - Elminti raccolti dal Dott. Elio Modigliani alle isole Mentawei, Engano e Sumatia. Bollettino dei Musei di Zool. e Anat. compar. della $R$. Univ. di Genova, 1898, $\mathrm{n}^{\circ}$ 64, p. 1-23, pl. I, fig. 1-19, Id. in Annali del Museo civico... Genova, ser. $2^{\circ}$, XIX (XXIX, luglio 1898), p. 102-124, pl. I, fig. 1-19.

1926, Petrov (A.-M.). - La faune des vers parasites des carnivores domestiques du Turkestan. Travaux de l'Institut d'Etat de la Médecine Vétérinaire expérimentale, II, livr. 2, Moscou, 1926 ; tirage à part, 14 pages.

1924, Popov (N.-P.). - Les vers parasites des carnivores domestiques d'Arménie. Travaux de l'Institut Tropical d'Arménie, I, Moscou, 1924. Tirage à part, p. 3-8.

1819, Runolphi (C.-A.). - Entozoorum Synopsis cui accedunt mantissa duplex et indices locupletissimi. Berolini, $1819, \mathrm{x}+811$ p., pl. I-III.

1933, SANDGROUND (J.-H.). - Report on the nematode parasites collected by the Kelley-Roosevelts expedition to Indo-China with description of several new species. Part. 1. Parasites of birds. Part. 2. Parasites of mammals. Zeitschrift für Parasitenkunde, V, Heft 3/4, 20-5-1933, p. 542-583, fig. 1-33.

1933, - Reports on the scientific results of an expedition to the southwestern Highlands of Tanganyika Territory. VI. Parasitic Nematodes from East Africa and Southern Rhodesia. Bull. Museum Comparative Zoölogy at Havard College, LXXV, $\mathrm{n}^{\circ}$ 6, aug. 1933, p. 263-293, fig. 1-14.

1935, - Spirura michiganensis n. sp. and Rictularia halli n. sp., two new parasitic nematodes from Eutamias striatus lysteri (Richardson). Transact. American Microscopical Society, LIV, $\mathrm{n}^{\circ}$ 2, april 1935, p. 155-166, pl. XXVIII, fig. 1-9.

1927, Schulz (R.-E.). - Zur Kenntnis der Helminthenfauna der Nagetiere der Union S.S.R. II. Spirurata Raill. et Henry, 1914. Travaux de l'Institut d'Etat de la Médecine Vétérinaire Expérimentale. Moscou, IV, livr. 2, 1927, p. 36-65, fig. 1-20.

1925, Schwartz (B.). - Parasitic Nematodes from Tonkin, Indo-China, including a new specie of Ascaridia. Proceed. Un. St. Nat. Museum, $\mathrm{n}^{\circ} 2538$, LXVI, art. 1, 1925, p. 1-9, fig. 1 .

1915, Seurat (L.-G.). - Sur les Rictulaires des Carnivores du Nord-Africain et les affinités du genre Rictularia. C.R. Soc. Biol., LXXVIII, n 11, 25-6-1915, p. 318-322, fig. 1-3.

1916, - Sur l'habitat normal et les affinités du Rictularia proni Seur. C.R. Soc. Biol., LXXIX, n 3, févr. 1916, p. 146-149, fig. 1-2.

1917, - Sur les Spiroptères des Carnivores du Nord-Africain. Bull. Soc. Histoire Naturelle Afrique du Nord, VIII, n 1, 15-1-1917, p. 21-24.

1930, - Nématodes. Exploration zoologique de l'Algérie de 1830 à 1930. Chapitre VII, p. 555-566, fig. texte 23-26, pl. XVI, fig. 1-2. Collection du Centenaire de l'Algérie. Paris, Masson et Cie, édit., 1930.

1916, - Sur un nouveau Dispharage des Palmipèdes. C.R. Soc. Biol., LXXIX, $\mathrm{n}^{\circ} 15,29-7-1916$, p. 785-788, fig. 1-5.

1916, Skrjabin (K.-J.). - Seuratia N.G. nouveau genre de Nématodes d'Oiseaux. C.R. Soc. Biol., LXXIX, $\mathrm{n}^{\circ}$ 18, 18-11-1916, p. 971-973.

Ann. de parasitologie, T. XX, $\mathrm{N}^{\text {os }} 1-2,1944-1945$. 
1924, Skrjabin (K.-I.) et Popov (N.-P.). - Bref aperçu sur l'activité de la mission helminthologique en Arménie pendant l'année 1923. Journal Russe de Médecine Tropicale. Moscou, I, n 2, 1924, p. 58-63.

1888 , Sonsino (P.). - Notizie elmintologiche. II. Rictularia plagiostoma e specie affini. Atti della Società Toscana di Scienze naturali. Processi verbali, VI, adunanza del 1-7-1888, p. 115-119.

1888, - Notices helminthologiques, II. Rictularia plagiostoma et espèces semblables. Arch. ital. de Biol., Turin, X, 1888, p. 192-196.

1930, SPREHN (K.). - Wichtige Helminthen einiger Laboratorium-Tiere. Tabula Biologice. Ed. W. Junk, VI, Supplementum, II, 1930, p. 244-276.

1919, Steiner (G.). - Untersuchungen über den allgemeinen Bauplan des Nematodenkörpers. Ein Beitrag zur Aufhellung des Stammesgeschichte und Verwandtschaftsverhältnisse der Nematoden. Zoologische Jahrbücher Abt. für Anat., XLIII, Heft 1, 6-12-1919, p. 1-96, 55 fig. texte (A-E2), pl. I-III, fig. 1-16.

1900, Srossich (M.). - Contributó allo studio degli Elminti. Bolletino della Soc. adriatica di Scienze naturali in Trieste, XX, 1900, p. 1-8, pl. I, fig. $1-8$; pl. II, fig. 9-18.

1928, Travassos (L.). - Sobre uma especie do genero Rictularia Frœlich (Nematoda). Bol. Biol. S. Panto, fasc. 14, 4-12-1928, p. 129-134, fig. 1-6.

1931, Tubangui (M.-A.). - Worm parasites of the brown rat (Mus norvegicus) in the Philippine Islands, with special reference to those forms that may be transmitted to human beings. The Philippire Journal of Science, XLVI, $\mathrm{n}^{\circ} 4$, dec. 1931 , p. 537-591, fig. $1 a-19 c$.

1938, Tupangui (M.-A.) et Masiluñgan (V.-A.). - Nematodes in the collection of the Philippine Bureau of Science, III. The Philippine Journal of Science, LXIV, 1937, $\mathrm{n}^{\circ} 3$, nov. 1938, p. 257-267, pl. I-III.

1861, WedL (C.). - Z Zur Helminthenfauna Egyptens (2te Abtheilung), III-V. Sitzungsber. der Kaiserl. Akad. der Wissenschaften Wien. Mathem. Naturwiss. Classe, XLIV, I Abt., Nr. 9, 1861, p. 463-482, pl. I-III, fig. 1-42.

1869, Willemoes-Suhm (R. von). - Helminthologische Notizen. Zeit. für wissensch. Zool, XIX, Heft 3, 6-9-1869, p. 469-475, pl. XXXV, fig. 1-5.

- Helminthologische Notizen, III. Zeitschrift für wissensch. Zoologie, XXIII, Heft 3, 18-11-1873, p. 331-345, pl. XVII, fig. 1-11.

1928, Witenberg (G.). - Reptilien als Zwischenwirte parasitischer Würmer von Katze und Hund. Tierärztliche Rundschau, XXXIV, 1928, Nr. 32, p. 603 .

1934, - Parasitic worms of dogs and cats in Palestine. The Veterinary Record, vol. XIV, $\mathrm{n}^{\circ}$ 9, 3-3-1934, p. 232-239.

1926, Yonke (W.) et Maplestone (P.-A.). - The Nematode Parasites of Vertebrates. London, 1926, $\mathrm{xx}+536$ p., fig. 1-307.

Institut de parasitologie de la Faculté de Médecine de Paris (Directeur: Prof. E. Brumpt) 